\title{
Poorly differentiated neuroendocrine carcinoma of the ovary-a case report
}

\section{Introduction}

Neuroendocrine tumors most commonly appear in the gastrointestinal tract or the lungs. This type of neoplasms is uncommon in the gynecologic tract and account for about $2 \%$ of all gynecological malignancies, but may be also metastatic from other sites. Large cell neuroendocrine carcinoma of the ovary, or ovarian undifferentiated non-small cell carcinoma of neuroendocrine type is rare and an aggressive tumor with tendency to present at advanced stages and to cause death after a short postoperative duration. Pathological diagnosis and immunohistochemistry (when distinguishing primary ovarian carcinomas from metastatic tumors) are crucial to guide therapy, but protocols for standard treatment are not yet established.

\section{Case report}

We report a case of 42-year old women, multipara, with no significant past medical history, who was referred to our Clinic with pain in lower part of abdomen, rapid weight loss and large tumorous pelvic mass, adjacent to right adnexa. After reception to the clinic, NMR scan was performed and showed large pelvis mass, para-aortal and para-illiacal lymphadenopathy, ascites, suspected metastatic deposits on liver and peritoneal carcinomatosis. Value of CA 125 was slightly elevated, while other tumor markers specific for ovarian carcinoma were in normal range. She underwent surgery (hysterectomy with bilateral adnexectomy, total omentectomy, appendectomy and bipolar colostomy).

Intraoperative finding confirmed presence of large pelvic and abdominal necrotic tumorous mass adjacent to right adnexa, surface metastatic deposits in sigmoid colon and omentum, conglomerate of para-aortal and parailliacal lymph nodes.

Histopathological examination revealed $745 \mathrm{~g}, 13 \times 13 \times 90 \mathrm{~mm}$ tumor origin of the right ovary, but was uncertain about type of malignant disease and additional immunohistochemistry was required. Postoperative recovery started regularly, her laboratory analyses were in normal range and discharge of the patient was planned. Ten days after primary surgery, her general condition started to getting worse, including thrombosis in superior vena cava diagnosed on the chest CT scan, pleural effusion, poor oxygen saturation, elevation of LDH (ten times higher than normal values) and thrombocyte levels. Hematologist was consulted immediately and he presented assumption that it is a case of neuroendocrine carcinoma and requested immediate finding of immunohistochemistry. Immunohistochemistry diagnosis was "poorly differentiated neuroendocrine carcinoma" of uncertain primary origin, with high mitotic index (32mitoses/10 HPF) and poor prognosis. Immunohistochemically, the tumor cells were positive for EMA and CAM 5.2, but the most consistent reactivity was for neuroendocrine tumor markers-Synaptophysin and NSE. Large areas of tumor necrosis were found. Twenty days after primary surgery, another laparotomy with partial ileal resection and terminal ileostomy was performed because of postoperative ileus, caused by multiple adhesions and partial necrosis in terminal ileum. Patient died within seven days from pulmonary embolism and cardiorespiratory
Volume 6 Issue 2 - 2017

\author{
Milena Mitrovic,' Vukajlovic S,' Argirovic R,' \\ Aksam S,' Uljarevic D,' Dokic M,' Milanovic \\ I' \\ 'Postoperative department, Clinical Center Serbia, Serbia \\ ${ }^{2}$ Minimally invasive surgery department, Clinical Center Serbia, \\ Serbia
}

Correspondence: Milena Mitrovic, Postoperative department Clinic of gynecology and obstetrics Clinical Center Serbia, Belgrade, Serbia Email milena.mitrovicdr@gmail.com

Received: June 25, 2016 | Published: February 24, 2017

insufficiency, despite continuous anticoagulant therapy and treatment in Intensive care department.

\section{Discussion}

Neuroendocrine carcinomas are heterogenous and specific group of neoplasms. Neuroendocrine differentiation may be found in a variety of primary ovarian tumors, including small-cell carcinoma of the pulmonary type, carcinoid tumor, Sertoli-Leydig cell tumors, teratomas and large-cell neuroendocrine carcinoma. Primary large-cell neuroendocrine carcinoma is histologically similar to neuroendocrine carcinomas of other organs and regarding this malignant disease several theories have been proposed, including the existence of primitive cells capable of differentiating into other cell types or activation of genes promoting neuroendocrine differentiation in non/neuroendocrine ovarian cells. The other possibility is that associated epithelial tumor or teratoma was overgrown by neuroendocrine carcinoma. There is no standard treatment for large cell neuroendocrine carcinoma of the ovary. Although it is very rare, we should always consider this type of ovarian carcinoma. According available reports from literature various combination chemotherapy regimens have been used in previous studies and survival periods varied among groups. Surgery is therapeutic choice for localised disease, but extent disease requires multidisciplinary approach and further future research in treatment. By presenting this case we also wanted to emphasize the importance of regular ultrasound examination; our patient was admitted to our hospital with disseminated malignant disease, but she was gynecologically examined only when abdominal pain became constant and unbearable. If diagnosed at earlier stage, prognosis could be better.

\section{Acknowledgments}

None.

\section{Conflicts of interest}

None. 
Table I Neuroendocrine Carcinoma of the Ovary- Clinical and Pathological Profile

\begin{tabular}{ll}
\hline Age & $\mathbf{4 2}$ \\
Chief Complaint & Abdominal pain, rapid weight loss \\
Serum Markers & CA I $2576 \mathrm{kU} / \mathrm{L}, \mathrm{CA}$ I9-9 I3 kU/L, \\
& CEA $2.2 \mathrm{mcg} / \mathrm{L}$ \\
& Total Abdominal Hysterectomy, Bilateral \\
& Salpingo-Oophorectomy, Bilateral \\
& Illiacal Lymph Node Dissection, Total \\
Surgery & Omentectomy, Appendectomy and \\
& Bipolar Colostomy \\
& Right ovary I3cm \\
Gross View & Large, Anaplastic cells \\
Cell Size & Large cell Neuroendocrine \\
Stage (Immunohostochemistry) & Carcinoma-G3, Unknown Primary \\
& Origin \\
Mitotic index & $32 / 10$ HPF, proliferation status Ki-67, \\
& index 60\% \\
Immunoreactivity & Synaptophysin, NSE 80\%, \\
Chromogranin<50\%, CD 56 20\%, & Calretinin,Vimentin \\
Chemotherapy & Absent \\
Outcome & Not performed \\
\hline
\end{tabular}

\section{References}

1. Reed NS, Gomez-Garcia E, Gallardo-Rincon D, et al. Gynecologic Cancer InterGroup (GCIG) consensus review for carcinoid tumors of the ovary. Int J Gynecol Cancer. 2014;24(9 Suppl 3):S35-S41.

2. Behnam K, Kabus D, Behnam M. Primary ovarian undifferentiated non-small cell carcinoma, neuroendocrine type. Gynecol Oncol. 2004;92(1):372-375.

3. Dundr P, Fischerová D, Povýsil C, et al. Primary pure largecell neuroendocrine carcinoma of the ovary. Pathol Res Pract. 2008;204(2):133-137.

4. Yasuda M, Kajiwara H, Osamura YR, et al. Ovarian carcinomas with neuroendocrine differentiation: Review of five cases reffering to immunohistochemical characterisation. J Obstet Gynaecol Res. 2006;32(4):387-395.

5. VerasE,DeaversMT,SilvaEG, etal.Ovariannonsmallcellneuroendocrine carcinoma: a clinicopathologic and immunohistochemical study of 11 cases. Am J Surg Pathol. 2007;31(5):774-782. 